\title{
Analysis on Monopole Antenna for Moisture Determination in Oil Palm Fruit Using Finite Difference Method
}

\author{
E. M. Cheng ${ }^{\dagger}$, Z. Abbas*, H. A. Rahim @ Samsuddin**, K. Y. Lee***, K. Y. You ${ }^{\S \S}$, \\ J. Hassan*, H. Zainuddin* and S.F. Khor ${ }^{\S}$
}

\begin{abstract}
Finite difference analysis were applied to study the principle operation of monopole antenna for moisture determination in oil palm fruit at $2 \mathrm{GHz}$. The electromagnetic field interact with oil palm fruit on the interface between the antenna and oil palm fruit and cause a reflection. The reflection measurement is based on mismatch impedance or dielectric properties between two media. Reflection coefficient is used to quantify the level of reflection. The monopole antenna was made of RG405/U semi-rigid coaxial cable with an inner and outer diameter of $0.45 \mathrm{~mm}$ and $1.50 \mathrm{~mm}$, respectively with $2.23 \mathrm{~mm}$ length of protruding conductor over $5.66 \mathrm{~cm}$ length of monopole antenna. This monopole antenna for moisture detection was compared with induced EMF method in terms of reflection coefficient at $2 \mathrm{GHz}$. The results show that the complex reflection coefficient measured using monopole antenna provides significant results to predict moisture content in oil palm fruit.
\end{abstract}

Keywords: Finite different method, Oil palm fruit, Moisture content, Monopole antenna

\section{Introduction}

Moisture content $(\mathrm{mc})$ of agricultural products is one of the most important parameters for determining quality of yield of agriculture. The optimum time for harvesting and potential for safe storage is required. It is also an important parameter in determining the market price because the moisture contents in agricultural products determine the value of the products. In the processed agricultural products e.g. grains for flour [1], other food products or animal feeds, moisture content in these materials is important information for efficient processing and achieving the desired highquality products.

Using standard oven drying methods [2] measuring moisture content in agricultural products require specific time periods at specific temperatures. Even though this method is accurate in determining moisture content in fruits, it is also tedious, time-consuming, and not suitable in the agro-production. Hence, a rapid testing microwave method need to be developed as proposed in this work.

In the twentieth century, microwave method was

$\dagger$ Corresponding Author: School of Mechatronic Engineering, Universiti Malaysia Perlis, Malaysia. (emcheng@unimap.edu.my)

* Faculty of Science, Dept. of Physics, Universiti Putra Malaysia, Malaysia. (\{za, jumiah, hishamuddin\}@science.upm.edu.my)

** School of Computer and Communication Engineering, Universiti Malaysia Perlis, Malaysia (haslizarahim@unimap.edu.my)

*** Dept. of Electrical and Electronic Engineering, Faculty of Engineering and Science, Tunku Abdul Rahman University, Malaysia. (kylee@ utar.edu.my)

§ School of Electrical System Engineering, Universiti Malaysia Perlis, Malaysia. (sfkhor@unimap.edu.my)

$\S \S$ Radio Communication Engineering Department, Faculty of Electrical Engineering, Universiti Teknologi Malaysia, Malaysia. (kyyou@fke.utm.my)

Received: August 28, 2015; Accepted: July 21, 2016 implemented in soil moisture detection [3], dehydration of fruit and heating [4].

In earlier times, many studies about the electrical resistance of vegetation have shown that electrical resistance is correlated with moisture content. The high correlation between permittivity of material and water content in the material hasten the usage of microwave method in sensing moisture content [5]. Hence, the principle where the dielectric property of the agricultural products is highly correlated with moisture content is proposed.

The dielectric properties of agricultural products is a function of moisture content, frequency of the applied electromagnetic field, temperature, density and structure of the materials $[6,7]$. When an external electric field is applied in fresh agricultural products (moist material), the polar water molecules in the material takes times to build up an equilibrium polarization. If high frequency of electric field strength is imposed, the polarization will lag behind the changing field. The dielectric properties of the material in time-dependent fields will therefore deviate from the corresponding equilibrium properties in the steady fields.

Currently, Malaysia has been the second largest producer of palm oil in the world. In 2011, the palm oil sector was the fourth largest contributor to Malaysia's economy, which contributes USD 16.8 billion to Malaysia's Gross National Income (GNI). Therefore, large efforts have been imposed to maintain or optimize this profitable industry, and finding the most accurate and efficient approach to optimize the yield (palm oil).

The amount of moisture content in oil palm fruit is one of the indicators to assess its ripeness. In other words, the moisture content can be used to determine the best 


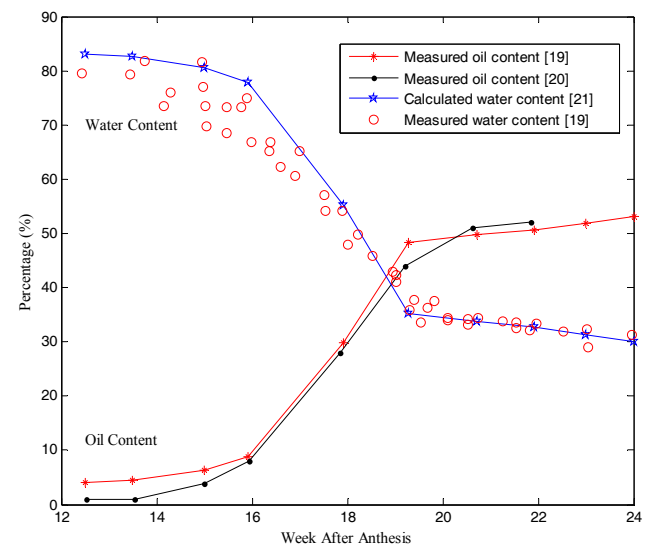

Fig. 1. Variation of water and oil content over week after anthesis

time to harvest oil palm fruit for oil extraction. Since oil palm fruit is the main commodity crop in Malaysia, a wrong judgement on the ripeness of oil palm fruit may cause severe economic losses. In this work, an innovative attempt has been worked out by using monopole antenna for moisture sensing in oil palm fruit to predict the best harvest time for oil palm fruit.

The determination of ripeness of oil palm fruit can be carried out via the measurement of moisture content in oil palm fruit. Ariffin et al. [8] reported that the moisture content in oil palm fruit is closely related with fruit ripeness. In Fig. 1, it can be observed that the moisture content is higher in fresh oil palm fruit than the ripe fruit at the early stage of fruit growth. The moisture content in the fruit declines progressively when the number of weeks after anthesis increases. It declines drastically to approximately $40 \%$ in the ripe fruit from week 16 to week 17 after anthesis. Moisture content declines insignificantly from week 18 to week 24 . The decline in moisture content is about the same rate as the accumulation of oil in the fruit. Hence, there is a close relationship between the moisture content and oil content in the fruit. This phenomenon is helpful to gauge the fruit ripeness. In order to develop a reliable and cost effective instrumentation system for ripeness of oil palm fruit, numerical method need to be implemented prior to instrumentation system development.

Finite difference method used in this work is a cost effective technique. Unlike the previous work using finite element method (FEM) on coaxial probe [9], finite difference method does not require commercial software to run finite element analysis. Finite difference computer program is simple and efficient way to calculate reflection coefficient of coaxial probe.

\section{Methodology}

\subsection{Monopole antenna}

In this work, monopole antenna was fabricated to

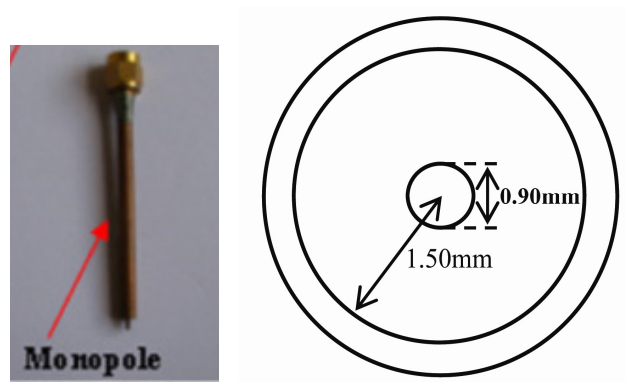

(a)

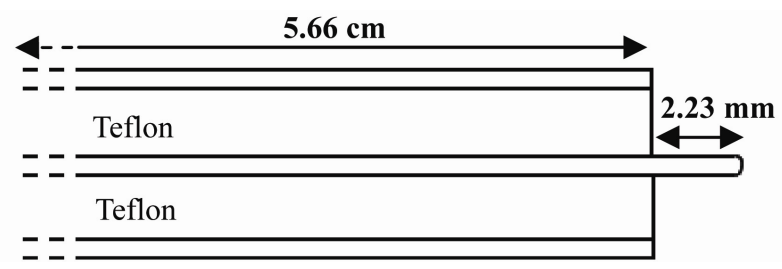

(b)

Fig. 2. (a) Cross section and (b) inner side view of monopole antenna

measure the reflection coefficient of oil palm fruits. The monopole antenna was fabricated of SMA (Sub Miniature version A) connector and RG405/U semi-rigid cable as shown in Fig. 2. Hard and malleable coaxial cable with a maximum operating frequency of $40 \mathrm{GHz}$ which is also known as semi-rigid cables is utilised. RG405/U semi-rigid cable is commercially obtained in reel length of $3 \mathrm{~cm}$, and can be formed easily to a monopole antenna with a small inner conductor diameter and adjustable protruding length. The RG405/U semi-rigid cable was considered primarily due to suitability of sensor's size for moisture measurement in the oil palm fruits at $2 \mathrm{GHz}$. The RG405 is constructed in semi-rigid form where the structure is approximately equally the same as the rigid cable. The size of the commercial rigid cable is bulky and lead to inconvenience in this work.

The Teflon dielectric in semi-rigid cable is enclosed by a solid silver-coated copper-clad steel conductor as the outer conductor. The inner conductor is of the same material as outer conductor. The radius of inner and outer conducted is $0.45 \mathrm{~mm}$ and $1.50 \mathrm{~mm}$, respectively as shown in Fig. 2 (a). Meanwhile, the length of coaxial line and protruding conductor is $5.66 \mathrm{~cm}$ and $2.23 \mathrm{~mm}$, respectively as shown in Fig. 2 (b).

The connected low loss coaxial cable with monopole antenna was calibrated using one port commercial calibration standards (match load, open and short) at the input plane of antenna (end of feeder cable). In other words, the input plane is calibrated before measurement, hence characteristic of measurement would not be affected.

The monopole antenna is connected to a computer controlled HP 8720B vector network analyzer (VNA) as shown in Fig. 3. 


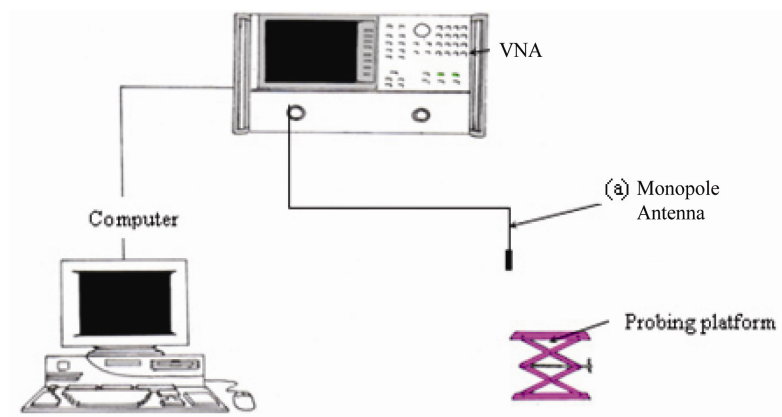

Fig. 3. Measurement setup

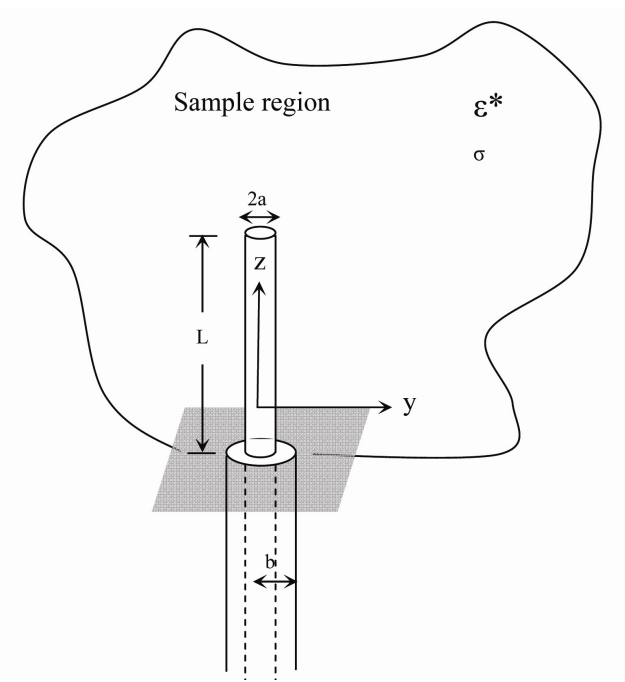

Fig.4. Geometry of coaxial line excited probe on a ground plane [13]

\subsection{Induced EMF method}

The induced EMF method is a classical method to compute the self and mutual impedances [10-12]. The induction of currents on adjacent and non-adjacent elements will be incurred upon mutual coupling effects between antennas, which may result in the array far-field pattern being altered compared with the ideal situation (mutual coupling is excluded). With the induced EMF approach, the electric field is produced in an antenna due to a known, predicted or calculated current distribution. This electric field is used to calculate the voltage induced in each elemental section of the antenna. To establish the voltage at the driving terminals of the antenna, the reciprocity theorem is required. This method can be used to determine antenna radiation resistance. In this work, the input impedance of medium, $Z_{\text {in }}$ which the monopole antenna interact with as shown in Fig. 4 can be expressed as Eq. (1) based on the induced EMF method [13].

$$
Z_{\text {in }}=j \frac{\eta}{4 \pi \sin ^{2}(k L)} \int_{0}^{L} \sin k(L-z) E(z) d z
$$

where

$$
\begin{gathered}
E(z)=\frac{e^{-j k r_{1}}}{r_{1}}+\frac{e^{-j k r_{2}}}{r_{2}}-2 \cos (k L) \frac{e^{-j k r}}{r} \\
r_{1}=\sqrt{(L-z)^{2}+a^{2}} \\
r_{2}=\sqrt{(L+z)^{2}+a^{2}} \\
r=\sqrt{a^{2}+z^{2}} \\
\eta=\sqrt{\mu_{0} / \varepsilon^{*}}=\frac{\omega \mu_{0}}{k} \\
k^{2}=\omega^{2} \mu_{0} \varepsilon^{*} \\
\varepsilon^{*}=\varepsilon_{0}\left(\varepsilon^{\prime}-j \frac{\sigma}{\omega \varepsilon_{0}}\right)
\end{gathered}
$$

where $\eta$ is intrinsic impedance, $\mathrm{L}$ is length of protruding conductor, $\mathrm{k}$ is phase constant, $\sigma$ is conductivity, $\varepsilon^{*}$ is complex permittivity, $\varepsilon^{\prime}$ is dielectric constant, $\omega$ is angular velocity and a is radius of protruding conductor. Meanwhile, $\varepsilon_{0}$ and $\mu_{0}$ is permittivity and permeability in free space, respectively. Hence, reflection coefficient, $\Gamma$ can be expressed as

$$
\Gamma=\frac{Z_{\text {in }}-Z_{0}}{Z_{\text {in }}+Z_{0}}
$$

where $Z_{0}$ is characteristic impedance of coaxial line, i.e. $50 \Omega$.

\subsection{Moisture measurement}

Moisture content was measured using standard oven method which proposed by Malaysia Palm Oil Board, MPOB (formerly called as Palm Oil Research Institute of Malaysia, PORIM) [2]. This method has been extensively used to determine the amount of true moisture content in oil palm fruits in oil palm industry. The mc in fruit is then calculated using the wet basic formula as in Eq. (10):

$$
\begin{aligned}
& \text { mc in fruit }= \\
& \frac{\text { Weight before dry-Weight after dry }}{\text { Weight before dry }} \times 100 \%
\end{aligned}
$$

The determined $\mathrm{mc}$ is important to calculate $\varepsilon^{*}$ using Eq. (22) and listed parameters in Table 1. $\varepsilon^{*}$ is one of the main parameter to calculate $\Gamma$ from induced EMF method and FDM.

\subsection{Finite Difference Method (FDM)}

In the computation work of FDM, iterative method was used to solve large system of simultaneous equations. Iterative method uses the calculated field in volt in matrix from previous iteration (input matrix) to calculate and overwrite matrix for next iteration (output matrix) until it 
converges to a correct value.

These field values are arranged to form a matrix. Field in matrix is applied to equation

$$
E_{i, j}=\frac{1}{4}\left(E_{i+1, j}+E_{i-1, j}+E_{i, j+1}+E_{i, j-1}\right)
$$

where $\mathrm{i}$ and $\mathrm{j}$ is sequence of element along vertical axis horizontal axis, respectively.

Plane $\mathrm{CD}$ is the boundary between two different materials, i.e., the Teflon in the coaxial line and the sample. On the dielectric boundary (Fig. 6), the boundary condition,

$$
\mathrm{D}_{1 \mathrm{n}}=\mathrm{D}_{2 \mathrm{n}}
$$

must be imposed where $D_{1 n}$ and $D_{2 n}$ are the normal components of the electric flux density at dielectric filler in coaxial line and in the sample being tested, respectively. This condition is based on Gauss' Law for electric fields, i.e.,

$$
\oint D \cdot d l=\oint \varepsilon * E \cdot d l=Q_{e n c}=0
$$

Since no free charge is deliberately placed on the dielectric boundary. Substituting $\mathrm{E}=-\nabla V$ in Eq. (13) gives:

$$
0=\oint \varepsilon * \nabla V \cdot d l=\oint \varepsilon * \frac{\partial V}{\partial n} \cdot d l
$$

where $\frac{\partial V}{\partial n}$ denotes the derivative of $\mathrm{E}$ normal to the contour l. Applying Eq. (14) to the interface in Fig. 7 yields

$$
E_{0}=\frac{\varepsilon_{1}}{2\left(\varepsilon_{1}+\varepsilon_{2}\right)} E_{1}+\frac{\varepsilon_{2}}{2\left(\varepsilon_{1}+\varepsilon_{2}\right)} E_{3}+\frac{1}{4} E_{2}+\frac{1}{4} E_{4}
$$

The finite difference potential results on plane $\mathrm{CD}$ in inset Fig. 6 (cross section area of aperture of coaxial line, $\mathrm{V})$ were computed. The potential difference, V, and the total charge, $\mathrm{Q}_{\text {total }}$, at the area of the aperture of the probe can be determined easily using Eq. (16) and (17), respectively [9].

$$
\begin{gathered}
V=\int_{a}^{b} E d \rho \\
Q_{\text {total }}=\varepsilon^{*} \int_{a}^{b} \int_{0}^{2 \pi} E \rho d \varphi d \rho
\end{gathered}
$$

where $\rho$ is the radius at aperture of the coaxial probe, a is the inner radius of the coaxial probe, and $b$ is the outer radius of the coaxial probe. Hence, capacitance, $\mathrm{C}$ can be calculated using

$$
C=\frac{Q_{\text {total }}}{V}
$$

The normalized and characteristic admittance are expressed as:

$$
\begin{gathered}
\tilde{Y}=\frac{j \omega C}{Y_{0}} \\
\tilde{Y}_{0}=\frac{2 \pi}{\sqrt{\frac{\mu_{0}}{\varepsilon_{0} \varepsilon_{c}}} \cdot \ln \left(\frac{b}{a}\right)},
\end{gathered}
$$

where $\varepsilon_{0}$ is the permittivity in free space, $\varepsilon_{c}=$ 2.05-j0.0005 (the relative permittivity of PTFE in coaxial line), and $\mu_{0}$ is the free space of permeability. The reflection coefficient, $\Gamma$ can be expressed as.

$$
\Gamma=\frac{1-\tilde{Y}}{1+\tilde{Y}}
$$

\section{Results and Discussions}

\subsection{Complex permittivity with moisture contents in oil palm fruit}

The variation in dielectric constant, $\varepsilon^{\prime}$ and loss factor, $\varepsilon^{\prime \prime}$ of oil palm fruit, with moisture contents, mc at $2 \mathrm{GHz}$ [9] is as shown in Fig. 5.

The abnormal behavior of $\varepsilon^{\prime}$ with mc below $40 \%$ is due to frequency relaxation of water at $2 \mathrm{GHz}$. In previous works [14,15], the $2 \mathrm{GHz}$ was studied and it has been suggested as an optimum frequency for monopole antenna in oil palm fruit moisture measurement. The bound water relaxation occurred below $2 \mathrm{GHz}$. Frequency cannot be less than $2 \mathrm{GHz}$ to avoid bound water relaxation frequency effect. In the meantime, microwave components at lower frequencies are cost effective for development of portable instrument in the future. The relationship between the mc

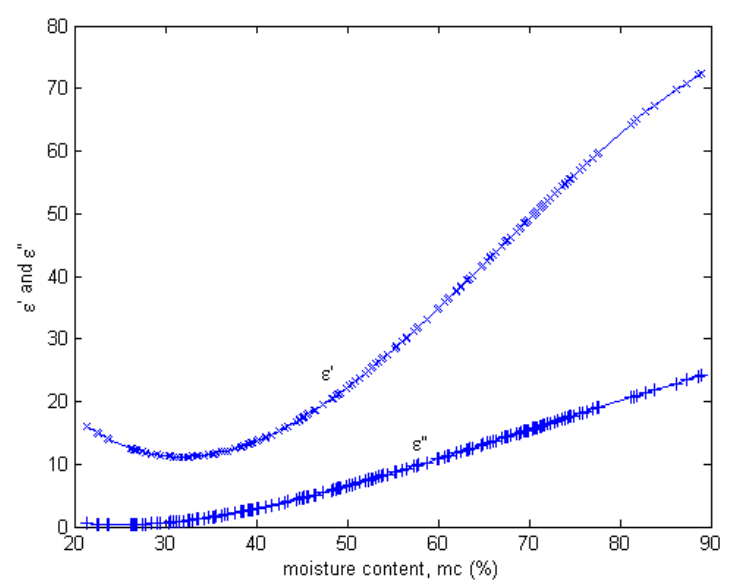

Fig. 5. Relationship between mc in oil palm fruit with $\varepsilon^{\prime}$ and $\varepsilon^{\prime \prime}$ at $2 \mathrm{GHz}$ 
Table 1. Description of parameters in Eq. (22)

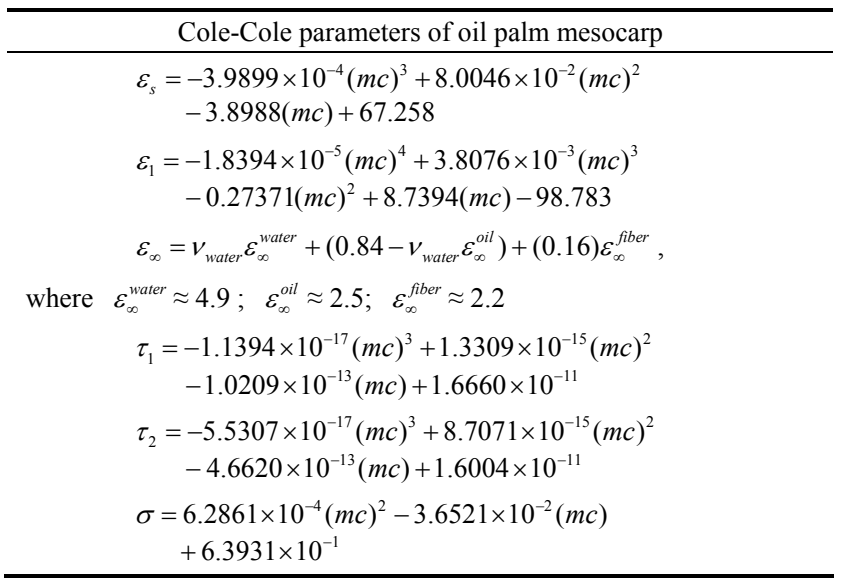

and oil accumulation is illustrated in Fig. 1 can be expressed as

$$
\varepsilon^{*}=\varepsilon_{\infty}+\frac{\varepsilon_{s}-\varepsilon_{1}}{1+j \omega \tau_{1}}+\frac{\varepsilon_{1}-\varepsilon_{\infty}}{1+j \omega \tau_{2}}-j \frac{\sigma}{\omega \varepsilon_{0}}
$$

The complex permittivity of sample under test, $\varepsilon^{*}$ are the functions of optical permittivity $\varepsilon_{\infty}$, static permittivity $\varepsilon_{s}$, Debye multi-dispersion formulation permittivity $\varepsilon_{1}$, angular velocity $\omega$, relaxation time for low frequency $\tau_{1}$, relaxation time for high frequency $\tau_{2}$, permittivity in free space, $\varepsilon_{0}$ and conductivity, $\sigma$ of the sample. The expression of these parameters is listed in Table 1 . It can be observed that all required parameters are a function of moisture [9]. The complex permittivity which is required for simulation and calculation of reflection coefficient were determined through Eq. (22) and parameter listed in Table 1.

Apart from effect due to frequency, mc in fruit is another factor which could determine occurrence of bound water effect. When moisture content is less than $40 \%$, the molecules of water are still bound with other substance in the fruit, e.g. fibre. Mobility of water molecules during the dielectric polarization is retarded as mc is less than $40 \%$. It leads to the decrement of dielectric constant and loss factor. When moisture content exceeds $40 \%$, sufficient free water molecule is presented in the fruit [9]. These free water molecules experience polarization and friction during the process of polarization in the applied field [16]. As a result, both dielectric constant and loss factor increase as moisture content exceeds $40 \%$.

Both $\varepsilon^{\prime}$ and $\varepsilon^{\prime \prime}$ are highly correlated with moisture content. The electromagnetic energy could be absorbed by water at microwave frequencies due to polarization and energy loss. In addition, the volume of moisture in the total volume of oil palm fruit influences the effective relative permittivity greatly. This is due to the relative permittivity of water $\left(\varepsilon^{\prime}=80\right.$ at DC stage $)$ is much greater than the other constituents in the materials, e.g. fiber fruits $\left(\varepsilon^{\prime}=2.2\right)$ and oil content : $\left(\varepsilon^{\prime}=2.5\right)[9]$.

\subsection{Magnitude of reflection coefficient}

The magnitude of reflection coefficient, $|\Gamma|$ that is acquired from induced EMF method does not show linear relationship between $20 \%$ to $90 \% \mathrm{mc}$. The magnitude decreases when the mc less than $40 \%$, and then it tends to become constant when the mc exceeds $40 \%$. The protruding conductor in monopole antenna has higher tendency to radiate. This tendency is strongly dependent on the length of the protruding conductor of monopole antenna and penetration depth in sample [9]. The monopole antenna propagates field from the protruding conductor and the field can be radiated farther.

The induced EMF method agrees with measured magnitude in region 1 . When mc exceeds $40 \%$ (region 2), the magnitude starts to rise and deviate from the measured magnitude. Radiation from the inner protruding conductor causes reflections from surface and object which is in the vicinity of the antenna, which will disturb the measurement. In order to avoid the disturbance in measurement, the monopole antenna should therefore be much shorter than one-tenth of the wavelength, $\lambda / 10$ in the fruit [9]. Short monopole antenna with length approximately or less than $\lambda / 10$ is good to be used in in-vivo measurement. When the length of protruding antenna is equal to $\lambda / 10$ or greater, a radiation field exists. In cases where the penetration depth in the fruit under study is greater than the fruit volume, it disturbs the measurement and additional errors are introduced in the measurement of the complex impedance in the sample. This is because the field is not contained within the sample only, but it also interacts with other objects outside the sample [17], [18].

FDM does not agree with measured magnitude initially in region 1 and it has better agreement with the measured magnitude in region 2 . The anomaly behaviour presented by induced EMF method can be explained through Fig. 5 where both dielectric constant and loss factor increase when moisture content exceeds $40 \%$. It heightens input impedance, $Z_{\text {in }}$ of monopole antenna. The mismatch impedance increases attributed to the response of input impedance, and in turn causes increment of $|\Gamma|$ when moisture content exceeds $40 \%$.

A full one-port calibration technique was implemented at the CD plane using commercial calibration standards kits (match load, open-circuit and short-circuit). CD plane is calibration plane, while $\mathrm{AB}$ plane is measurement plane. Under the assumption of quasi-TEM (transverse electromagnetic) mode, the measured reflection coefficient at $\mathrm{CD}$ plane of sample, $\Gamma_{C D}$ and $\mathrm{AB}$ plane, $\Gamma_{A B}$ can be determined using the term $e^{-j 2 k_{c} d_{c}}$, where $\mathrm{d}_{\mathrm{c}}$ and $\mathrm{k}_{\mathrm{c}}$ are the physical length (in meter) and propagation constant of coaxial line, respectively. The inconograph in Fig. 6 illustrate $\mathrm{AB}$ and $\mathrm{CD}$ plane. The measured $\Gamma_{C D}$ of the sample at the $\mathrm{CD}$ plane can be de-embedded to the 
Table 2. Mean magnitude error of reflection coefficient using FDM and induced EMF method

\begin{tabular}{c|c}
\hline Method & Mean magnitude error \\
\hline FDM (AB plane) & 0.0736 \\
\hline FDM (CD plane) & 0.0736 \\
\hline Induced EMF method (AB plane) & 0.0825 \\
\hline Induced EMF method (CD plane) & 0.0825 \\
\hline
\end{tabular}

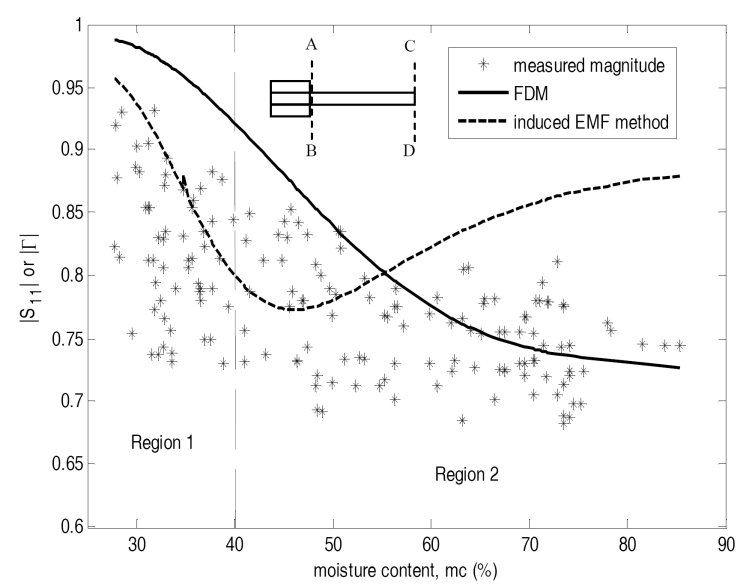

Fig. 6. A comparison of magnitude of reflection coefficient among measured data, FDM and induced EMF method.

connector end of the probe which coincide with the calibration $\mathrm{AB}$ plane to give an actual reflection coefficient, $\Gamma_{A B}$.

In Table 2, it can be observed that the magnitude of reflection coefficient of $\mathrm{FDM}$ in $\mathrm{AB}$ and $\mathrm{CD}$ plane is similar in mean magnitude error when compared to measured data. It means that the magnitude was unchanged when $\mathrm{CD}$ plane is extended to $\mathrm{AB}$ plane along the Teflonfilled monopole antenna. This is due to the lossless characteristics in Teflon. The loss factor from Teflon is about 0.0005 . Hence, the propagation of wave in lossless medium is not attenuated significantly. In other words, the attenuation constant, $\alpha=0 \mathrm{~Np} / \mathrm{m}$. Meanwhile, the induced EMF method shows mean magnitude error, 0.0825 for $\mathrm{mc}$ range $20 \%$ to $90 \%$.

In Fig. 6, it can be observed that magnitude of induced EMF method showed better agreement with measured data if compared with FDM when moisture content is less than $40 \%$ (region 1). The mean magnitude error for induced EMF method and FDM are 0.07 and 0.14 in region 1, respectively. Significant difference of mean magnitude error among the methods in region 1 can be observed. In region 1, the maximum oil content is accumulated. It is very important to predict $\mathrm{mc}$ accurately.

In the contrary, FDM shows better agreement with measured magnitude compared with induced EMF method when mc exceeds $40 \%$ (region 2) compared with induced EMF method. FDM and induced EMF method show different sign of gradient. The magnitude of FDM decreases from $40 \%$ to $90 \%$ of mc, whereas magnitude of

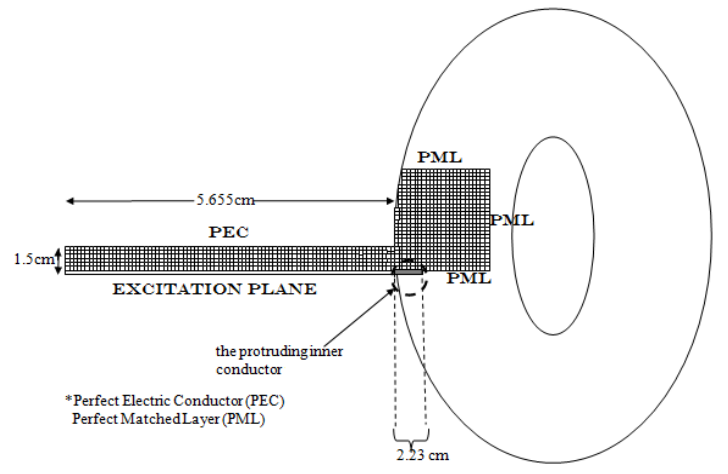

Fig. 7. Subdivide antenna and patch of oil palm fruit into mesh (solution region)

induced EMF method increased in region 2. The mean magnitude error obtained from FDM and induced EMF methods are 0.04 and 0.09 , respectively. Induced EMF method cannot be used to predict $\mathrm{mc}$ in this $\mathrm{mc}$ range because it shows positive gradient that is opposite with measured magnitude.

The fringing effect at the protruding conductor and aperture is due to the high conductivity of the moist media. Excessive $\mathrm{mc}(\mathrm{mc}>40 \%)$ causes high conductivity. FDM divide the electromagnetic analysis on the coaxial line and protruding antenna in mesh. If the number of developed mesh is sufficient enough as shown in Fig. 7, the fringing effect can be accurately considered. In addition, the aspect ratio of coaxial line, $b / a$ is also a major factor that describes the fringing effect. This factor is considered too in FDM. Thus, the FDM result has better agreement with measurement. However, the principle of induced EMF method does not take aspect ratio into account, but only the dimension of protruding conductor. The fringing field from aperture of coaxial line is not considered, but only far field. In addition, induced EMF method assumes uniform shape of the fruit. As a result, the assumption that is made in induced EMF method cause the severe discrepancy between calculated and measured result when moisture content exceeds $40 \%$.

\subsection{Phase of reflection coefficient}

The variation of phase with $\mathrm{mc}$ is shown in Fig. 8. The phase shift, $\varphi_{S 11}$ is determined by the physical length. It is illustrated in Fig. 9.

The extension from measurement plane (CD plane) to calibration plane (AB plane) causes the results in Fig. 6 to agree with measured results. The length of coaxial line is estimated between $6.30 \mathrm{~cm}$ and $6.40 \mathrm{~cm}$. When the physical length is varied, the optimum length of monopole antenna that provides the smallest error is slightly different with exact length, i.e. $5.66 \mathrm{~cm}$. The discrepancy of optimum length from exact length can be regarded as compensation for measurement errors. The optimum length of monopole antenna is longer than exact length by about $0.64 \mathrm{~cm}$. 
Table 3. Comparison of phase of FDM with induced EMF method and measured data in terms of phase of reflection coefficient

\begin{tabular}{c|c}
\hline Method & Mean phase error \\
\hline FDM (AB plane) & 8.27 degree \\
\hline FDM (CD plane) & 80.38 degree \\
\hline Induced EMF method (AB plane) & 87.09 degree \\
\hline Induced EMF method (CD plane) & 9.25 degree \\
\hline
\end{tabular}

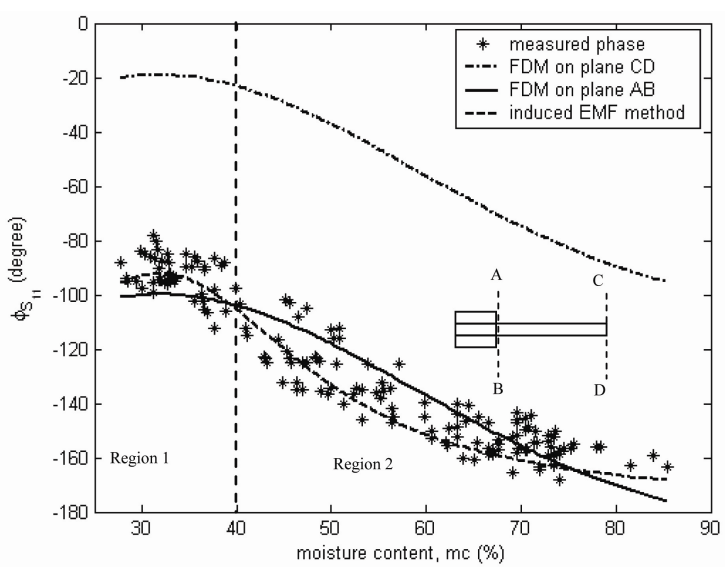

Fig. 8. A comparison of phase of reflection coefficient among measured data, finite difference method and induced EMF method.

Meanwhile, the wavelength range of applied field from $20 \%$ to $90 \%$ mc range is from $0.0018 \mathrm{~cm}$ to $0.0037 \mathrm{~cm}$ due to variation of travel speed of applied field.

The phase of reflection coefficient from induced EMF (solid line in Fig. 8) agrees slightly with measured data compared with FDM at the AB plane. FDM on AB plane and induced EMF method show similar mean phase errors, namely 8.27 degrees and 7.02 degrees respectively. The error shown by FDM is 80.38 degrees on the CD plane. After the plane is extended from $\mathrm{CD}$ plane (measurement plane) to $\mathrm{AB}$ (calibration plane), the error reduced to 8.27 degrees (as listed in Table 3 ). The measurement plane must coincide with the calibration plane to ensure consistency between FDM and measurement.

In Fig. 8, it can be observed that the phase of induced EMF method shows better agreement with the measured phase compared with FDM when range of mc is less than $40 \%$ (region 1). The mean phase error of induced EMF method is 5.57 degrees. FDM showed larger mean phase error on CD plane at 73.68 degrees compared with induced EMF method and FDM on AB plane. Even though the mean phase error of FDM reduces to 8.82 degrees after plane extension, the phase of induced EMF method still works better than FDM in region 1 (Fig. 8).

The FDM still shows the large mean phase error compared with induced EMF method on measurement plane (CD plane) in region 2 of Fig. 8. The mean phase error for induced EMF method in region 2 is 7.71 degrees.
Table 4. Relationship among moisture content, magnitude and phase of reflection coefficient

\begin{tabular}{|c|c|c|}
\hline Method & & Empirical/trendline equation \\
\hline \multirow{2}{*}{ Measurement } & Magnitude & $m c=-184.26(|\Gamma|)+196.84$ \\
\hline & Phase & $\mathrm{mc}=3.9 \times 10^{-3} \varphi^{2}+0.4017 \varphi+37.562$ \\
\hline \multirow[t]{2}{*}{ FDM } & Magnitude & $\begin{aligned} \mathrm{mc}= & -5908.70(|\Gamma|)^{3}+15538.00(|\Gamma|)^{2} \\
& -13706.00(|\Gamma|)+4100.60\end{aligned}$ \\
\hline & Phase & $\mathrm{mc}=-0.62 \varphi-26.51$ \\
\hline $\begin{array}{l}\text { Induced EMF } \\
\text { method }\end{array}$ & Phase & $\mathrm{mc}=-0.56 \varphi-20.76$ \\
\hline
\end{tabular}

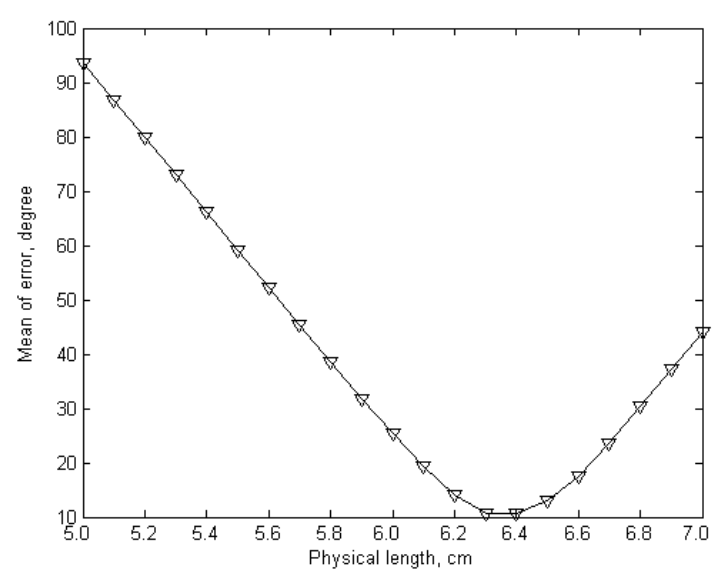

Fig. 9. Physical length of coaxial line (monopole antenna) with its phase error of reflection coefficient

Meanwhile, the mean phase error for FDM is 75.05 degrees. After plane extension, the mean phase error of FDM reduces to 7.82 degrees. This implies that the coinciding of measurement plane and calibration plane improves the phase of FDM by reducing the mean phase error about 67.23 degrees. Overall, FDM exhibits the least mean phase error, i.e. 8.27 degrees for mc from $20 \%$ to $90 \%$. In other words, FDM has better agreement with phase.

The moisture content can be determined from empirical equations listed in Table 4 in terms of magnitude and phase of reflection coefficient for each method, except induced EMF method because moisture empirical equation in terms of magnitude cannot be a mathematical function due to its failure in horizontal line test in Fig. 6. It implies that for every value of moisture content in the function of $|\Gamma|$ from induced EMF method, there is not one unique $|\Gamma|$.

The developed empirical equation for mc prediction is acquired through measured magnitude. $|\Gamma|$ and phase, $\phi$ over mc. Gaussian Elimination method was implemented to determine coefficients for empirical equations in Table 3 and Table 4 in terms of $|\Gamma|$ and $\phi$.

\section{Conclusion}

In this work, a monopole antenna which was made of RG405/U semi-rigid coaxial cable was used for moisture detection in oil palm fruit in conjunction with HP $8720 \mathrm{~B}$ 
vector network analyzer. Finite difference method and induced EMF method were used to compare with measured reflection coefficient in terms of magnitude and phase. It can be observed that finite difference method shows better agreement with measured magnitude of reflection coefficient with mean magnitude error of 0.0736. After transmission line method is conducted to extend CD plane to AB plane, mean phase error presented by FDM has been improved to 8.27 degrees if compared with induced EMF method which exhibit mean phase error of 9.25 degrees. FDM shows good agreement with measured phase of reflection coefficient, $\varphi_{S 11}$ or $\varphi$.

\section{Acknowledgements}

The authors wish to thank Universiti Putra Malaysia for providing financial support and materials for the research. Contribution from Universiti Malaysia Perlis (UniMAP) is highly appreciated in sponsorship under SLAB/SLAI Scheme.

\section{References}

[1] Nelson, S. O., Kraszewski, A. W., Trabelsi, S. and Kurt, C. Lawrence, "Using Cereal Grain Permittivity for Sensing Moisture Content," IEEE Trans. Instrum. Meas, vol. 49, no. 3, pp. 237-242, 1999.

[2] Siew, W.L., Tang, T.S., and Tan, T.A., PORIM Test Method, p4.2. Palm Oil Research Institute of Malaysia, Bangi, 1995, p.112-113.

[3] Schmugge, T. J., "Remote Sensing of Soil Moisture: Recent Advances," IEEE Trans. on Geoscience and Remote Sensing, Vol. GE-21, no. 3, pp. 336-344, July 1983.

[4] Khamis N.H.H., Ramli S., Ean T.C., Bakar W.Z.A.W., "Application of microwave technology for home industry," Asia-Pacific Conference on Applied Electromagnetics (APACE), p. 20-21, 2005.

[5] Kraszewski A.W., "Microwave aquametry - needs and perspectives," IEEE Trans. on Microwave Theory Techn., vol. MTT-39, no. 5, pp. 828-835, 1991.

[6] Kraszewski, A.W. and Nelson, S.O., "Application of microwave techniques in agricultural research," in Proceedings Microwave and Optoelectronics Conference SBMO/IEEE MTT-S International Vol. 1, pp. 117-126, 1995.

[7] Kraszewski, A.W. and Nelson, S.O, "Microwave Permittivity Determination in Agricultural Products," 12th International Conference on Microwaves and Radar (MIKON '98), Vol. 4, p. 230-242, May 1998.

[8] Ariffin, A. 2005. Fundamental aspects of oil and fats. In improving oil extraction rates in palm oil mills. Sabah: CEPP and UTM.

[9] Kok Yeow You and Zulkifly Abbas, Open-Ended
Coaxial Sensor Handbook: Formulations, Microwave Measurements and Applications: Lambert Academic Publishing, 2010.

[10] Burgess, R. E. 1944. Aerial Characteristics. Wireless Engr., vol. 21, pp. 154-160

[11] Pistolkors, A. A. 1929. The Radiation Resistance of Beam Antennas," Proc. IRE, vol. 17, pp. 562-579.

[12] King, H. E. 1957. Mutual Impedance of Unequal Length Antennas in Echelon. IRE, Trans. Antennas Propagat., Vol. AP-5, pp. 306-313.

[13] Misra, D. 1988. In Situ Electric Probe Method for Determining the Dielectric Properties of Materials, IEEE Trans. Instrum. Meas. vol 37, no 1, pp 157-160.

[14] Zulkifly Abbas, You Kok Yeow, Abdul Halim S haari, Azmi Zakaria, Jumiah Hassan, Kaida Khali $\mathrm{d}$ and Elias Saion, "Fast and Accurate Technique for Determination of Moisture Content in Oil Pal m Fruits using Open-Ended Coaxial Sensor," Jap anese Journal of Applied Physics, Vol. 44, No. 7 A, pp. 5272-5274, 2005.

[15] Lee Kim Yee, Zulkifly Abbas, Nur Sharizan Mohamed Dan, You Kok Yeow, "Portable Microwave Instrumentation System for Determination of Moisture Content in Oil Palm Fruits," Japanese Journal of Applied Physics, 48, 120219, pp. 1-3, 2009.

[16] Nyfros, E., and Vainikainen, P., Industrial Microwave Sensors, Artech House Inc., Norwood, MA., 1989.

[17] Burdette, E.C., Clain, F. L., Seals, J., "In Vivo Probe Measurement Technique for Determining Dielectric Properties at VHF Through Microwave Frequencies." IEEE Trans. \& Microwave Theory Tech., vol. MTT28, No. 4, pp. 414-427, 1980.

[18] Chen, L.F., Microwave Electronics: Measurement and Materials and Characterisation, John Wiley \& Sons Inc., p. 154-155, 2004.

[19] Abbas, Z. 1994. A microstrip sensor for determination of harvesting time for oil palm fruits. Master's Thesis, University of Agriculture Malaysia (UPM).

[20] Ariffin, A., Mat S.R., Banjari, M., and Wan, O.W.E. 1990. Morphological changes of the cellular component of the developing palm fruit (Tenera: Elaeis Guineesis). Palm Oil Research Institute of Malaysia Bulletin. 21:30-34.

[21] Hartley, C.W.S. 1988. The Oil Palm. $3^{\text {rd }}$ ed. Longman, London.

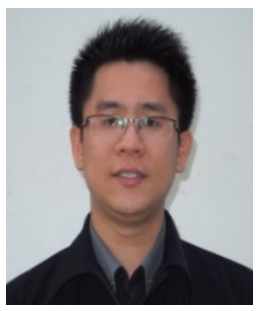

Ee Meng Cheng was born in 1980. He obtained his B.Sc (Honours) - Instrumentation Science in Universiti Putra Malaysia in 2004. He pursued his M. Sc. in Wave Propagation at the Institute for Mathematical Research on 2005 in Universiti Putra Malaysia and his Ph.D in Microwave at the Faculty of Science 
in 2007 at Universiti Putra Malaysia. Recently, he is senior lecturer in School of Mechatronic Engineering, Universiti Malaysia Perlis. His main personal research interest is in the computational electromagnetic modeling, microwave dielectric spectroscopy, wave propagation in RF \& microwave and microwave sensors development for food and agricultural applications.

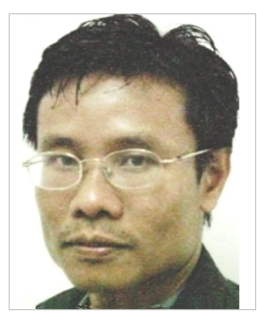

Zulkifly Abbas was born in Alor Setar, Malaysia, in 1962. He received the B.Sc. degree with honors in physics from the University of Malaysia, Kuala Lumpur, in 1986, the M.Sc. degree in microwave instrumentation from the Universiti Putra Malaysia (UPM), Serdang, in 1994, and the Ph.D. degree in electronic and electrical engineering from the University of Leeds, Leeds, U.K., in 2000. He is currently Associate Professor with the Department of Physics, UPM, where he has been a faculty member since 1987. His main personal research interest is in the theory, simulation, and instrumentation of electromagnetic wave propagation at microwave frequencies focusing on the development of microwave sensors for agricultural applications.

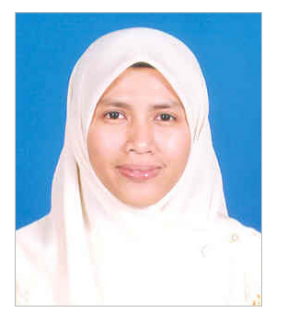

Hasliza A Rahim@ Samsuddin obtained her B.Sc. in Electrical Engineering (Modern Communication System) from University of Southern California, USA. She pursued her M.Sc. (Eng) at Universiti Sains Malaysia and $\mathrm{PhD}$ in Communication Engineering on 2015 at Universiti Malaysia Perlis.

Recently, she is senior lecturer in School of Computer and Communication Engineering, Universiti Malaysia Perlis. Her main personal research interest is in antenna design and bioelectromagnetism.

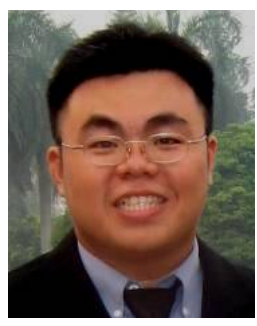

Kim Yee Lee was born in Muar, Johor, Malaysia. He received his BSc. Physics, MSc. (Microwaves), and PhD. (Microwaves) from Universiti Putra Malaysia in year 2002, 2004, and 2008 respectively. In December 2007, $\mathrm{He}$ joined Universiti Tunku Abdul Rahman as a Lecturer in Department of Electronics and Electrical Engineering. His areas of research include microwave measurement technique, microwave circuit and instrumentation, control and automation, material properties measurement, and instrumentation calibration.

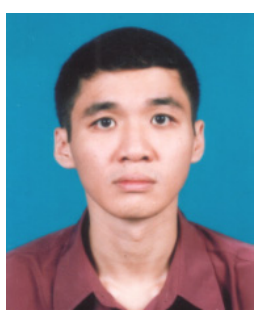

Kok Yeow You was born in 1977. He obtained his B.Sc Physics (Honours) degree in Universiti Kebangsaan Malaysia in 2001. He pursued his M. Sc. in Microwave at the Faculty of Science in 2003 and his Ph.D in Wave Propagation at the Institute for Mathematical Research in 2006 at Universiti

Putra Malaysia. Recently, he is senior lecturer at Radio Communication Engineering Department, Universiti Teknologi Malaysia. His main personal research interest is in the theory, simulation, and instrumentation of electromagnetic wave propagation at microwave frequencies focusing on the development of microwave sensors for agricultural applications.

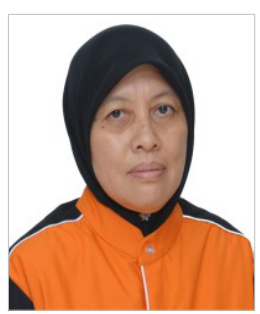

Jumiah Hassan is currently Associate Professor with the Department of Physics, Faculty of Science, UPM. Her main research interests include dielectric physics, materials science, and condensed matter physics.

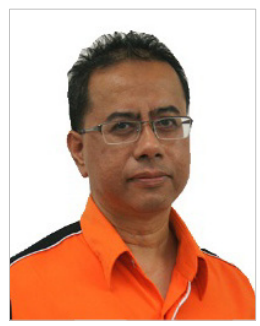

Hishamuddin Zainuddin is currently Associate Professor with the Department of Physics, Faculty of Science, UPM. His main research interests include Theoretical Physics, Quantum Physics, Geometry and Topology.

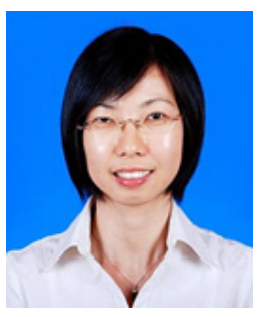

Shing Fhan Khor was born in 1982. She obtained her B. Sc. with Edu. (Honours)-Physics in Universiti Putra Malaysia in 2007. She pursued her $\mathrm{PhD}$ in Materials Science at the Faculty of Science in 2011 at Universiti Putra Malaysia. Recently, she is senior lecturer at School of Electrical Systems

Engineering, Universiti Malaysia Perlis (UniMAP). Her main personal research interest is in the glass science and focusing on dielectric, optical, mechanical and thermal properties. 